\title{
THE METHOD OF BALANCING THE PRODUCTION AND CONSUMPTION MODEL IN THE CASE OF INDIVISIBLE ARTICLES
}

\author{
Marek Ladyga, Maciej Tkacz \\ Institute of Mathematics, Czestochowa University of Technology \\ Częstochowa, Poland \\ marekladyga@im.pcz.pl,maciej.tkacz@im.pcz.pl
}

\begin{abstract}
In this article, a detailed case of the unbalanced production-consumption, namely a model, which is used in the case of indivisible articles, is presented. Also, the method of balancing this model is given, relying on introducing a certain ordering relation in the consumer's set.
\end{abstract}

Keywords: balancing, model, indivisible articles

\section{Introduction}

In article [1] the unsustainable production and consumption model was defined, in which occur $n \geq 2$ contractors, $m \geq 1$ producers and $n-m \geq 1$ consumers of a certain good. The model assumes that total demand exceeds total supply in a fixed period of time, what we write in the form of so-called uncorrected $n$-dimensional supply-demand vector, fulfilling the conditions

1) $p_{i}>0$ for every $i<m$

2) $p_{i}<0$ for every $m+1 \leq i \leq n$

3) $\sum_{i=1}^{n} p_{i}<0$

The general case was discussed in [1-4] articles. A detailed case are so-called indivisible articles, for example: washing machines, television sets etc. (you cannot buy 1.5 washing machine).

In this case, instead of uncorrected supply-demand vector, we consider so called discrete vector, ie. uncorrected supply-demand vector, of which every coordinate is an integer. 
Let there be vectors:

a) discrete vector $\overrightarrow{\mathbf{p}}=\left(p_{1}, \ldots, p_{n}\right)$, where $p_{i}>0$ for $i=1,2, \ldots m<n$ ( $p_{i}$ - demand of $i^{\text {th }}$ contractor), $p_{j}<0$ for $j=m+1, \ldots n$ ( $p_{j}$ - supply of $j^{\text {th }}$ contractor), so that $p_{1}+p_{2}+\ldots+p_{n}<0$,

b) maximum concession vector $\overrightarrow{\mathbf{u}}=\left(u_{1}, \ldots u_{n}\right)$, where $\left(u_{i} \in \mathrm{N} \cup\{0\}, u_{i}\right.$ - the size of the maximum possible concession of $i^{\text {th }}$ contractor), so that $\left(p_{1}+u_{1}\right)+\left(p_{2}+u_{2}\right)+\ldots+\left(p_{n}+u_{n}\right) \geq 0$,

c) vector $\overrightarrow{\mathbf{a}}=\left(a_{1}, a_{2}, \ldots, a_{n}\right), a_{i}$ - concession weight of $i^{\text {th }}$ contractor.

Taking $\overrightarrow{\mathbf{p}}^{\mathbf{0}}=\mathbf{p}, \overrightarrow{\mathbf{a}}^{0}=\overrightarrow{\mathbf{a}}$ for $s \geq 1$ we define the size of

$$
\begin{gathered}
r^{s}=-\left(p_{1}^{s-1}+\ldots+p_{n}^{s-1}\right) \\
N^{s}=\left\{i \leq n: \sum_{j \leq s} a_{i}^{j-1} r^{j} \leq u_{i}\right\} \\
\overrightarrow{\mathbf{a}}^{s}=\left(a_{1}^{s}, \ldots, a_{n}^{s}\right) \text { where } a_{i}^{s}= \begin{cases}0 & \text { for } i \notin N^{s} \\
a_{i}^{s-1}\left(B^{s-1}\right)^{-1} & \text { for } i \in N^{s}\end{cases}
\end{gathered}
$$

where $B^{s-1}=\sum_{j \in N^{s-1}} a_{j}^{s-1}$

$$
\begin{aligned}
& \bar{N}^{s}=N^{s-1} \backslash N^{s} \text { where } N^{o}=\{1, \ldots, n\} \\
& \overrightarrow{\mathbf{p}}^{s}=\left(p_{1}^{s}, \ldots, p_{n}^{s}\right) \text { where } p_{i}^{s}= \begin{cases}p_{i}^{s-1}+r^{s} \cdot a_{i}^{s-1} & \text { for } i \in N^{s} \\
p_{i}+u_{i} & \text { for } i \in \bar{N}^{s} \\
p_{i}^{s-1} & \text { for } i \in \cup_{j<s} \bar{N}^{j}\end{cases}
\end{aligned}
$$

The iteration process, whose properties were described in [2], we finish for $s^{\text {th }}$ iteration, such that $r^{s}=0$. Then vector $\overrightarrow{\mathbf{p}}^{s-1}$ balances the model. Let $\overrightarrow{\mathbf{x}}=\left(x_{1}, \ldots, x_{n}\right)$ be any vector. By $\mathrm{E}(\overrightarrow{\mathbf{x}})$ we understand vector $\left[\mathrm{E}\left(x_{1}\right), \ldots, \mathrm{E}\left(x_{n}\right)\right]$ where $\mathrm{E}\left(x_{i}\right)$ - integer's total part $x_{i}$. Because $\overrightarrow{\mathbf{p}}^{s-1}=\overrightarrow{\mathbf{p}}^{s}$, continuing in this article, by the overall vector correction vector $\overrightarrow{\mathbf{p}}^{0}$ we adopt vector $\overrightarrow{\mathbf{p}}^{s}$. 
If $\mathrm{E}\left(p_{1}^{s}\right)+\mathrm{E}\left(p_{2}^{s}\right)+\ldots+\mathrm{E}\left(p_{n}^{s}\right)=0$ then vector $\mathrm{E}\left(\overrightarrow{\mathrm{p}}^{s}\right)$ balances the model in case of indivisible articles. Assume that $t=\mathrm{E}\left(p_{1}^{s}\right)+\ldots+\mathrm{E}\left(p_{n}^{s}\right)>0$, which means that supply on certain good exceeds demand. In order to achieve sustainable modem, some producers should reduce the size of production (supply reduction) or, chosen consumers increase demand. In this situation, you may prefer consumer and use a second variant of balancing. It therefore remains to determine which consumer and by how much the demand should be increased. The solution can be as follows: add by one unit of good for those consumers who the most resigned.

\section{The balancing of model}

It is, therefore necessary to prove that:

a) has remained integer of units goods to distribute among consumers,

b) the number of consumers, which can increase demand by one unit of good (in this case, the border in output demand), is not smaller than number of units of goods, which is left to distribute.

It will be proved by the statement below.

\section{Statement 1.}

If:

$M_{1}=\left\{i \in N^{0}: m<i \leq n\right.$ and $\left.\mathrm{E}\left(p_{i}^{s}\right)-1 \geq p_{i}\right\}$ and $t>0$ then the cardinality of the set $M_{1}$ is bigger than $t$ and $t$ is an integer.

Proof.

$t=\sum_{i=1}^{n} E\left(p_{i}^{s}\right)$ is, obviously, an integer as a sum of integers.

For every $i \leq m$ there is an inequality of $p_{i}^{s} \geq 0$, or $E\left(p_{i}^{s}\right)-p_{i}^{s} \leq 0$, and for every $i>m$ there is an inequality $p_{i}^{s} \leq 0$, or $0 \leq E\left(p_{i}^{s}\right)-p_{i}^{s}<1$. Therefore:

$$
\begin{gathered}
t=\sum_{i=1}^{n} E\left(p_{i}^{s}\right)=\sum_{i=1}^{n} E\left(p_{i}^{s}\right)-\sum_{i=1}^{n} p_{i}^{s}= \\
=\sum_{i=1}^{m}\left(E\left(p_{i}^{s}\right)-p_{i}^{s}\right)+\sum_{i=m+1}^{n}\left(E\left(p_{i}^{s}\right)-p_{i}^{s}\right)<\sum_{i=m+1}^{n}\left(E\left(p_{i}^{s}\right)-p_{i}^{s}\right) \leq \# \bar{M}_{1}
\end{gathered}
$$

where: $\bar{M}_{1}=\left\{i \in N^{0}: m<i \leq n\right.$ and $\left.E\left(p_{i}^{s}\right) \neq p_{i}^{s}\right\}$. 
It remains to be presented, that $\bar{M}_{1} \subset M_{1}$.

Let $i \in \bar{M}_{1}$, or $E\left(p_{i}^{s}\right) \neq p_{i}^{s}, p_{i}^{s} \geq p_{i}^{0}$, therefore:

$p_{i}^{0} \leq p_{i}^{s}<E\left(p_{i}^{s}\right)$, or $p_{i}^{0}<E\left(p_{i}^{s}\right)$, but $p_{i}^{0}, E\left(p_{i}^{s}\right)$ belong to the set of integers, so there is inequality $p_{i}^{o} \leq E\left(p_{i}^{s}\right)-1$, therefore $i \in M_{1}$.

If $\bar{M}_{1} \subset M_{1}$, that $\# \overline{\mathrm{M}}_{1} \leq \# \mathrm{M}_{1}$ but $\# \overline{\mathrm{M}}_{1}>t$, hence $\# \mathrm{M}_{1}>t\left(\# \mathrm{M}_{1}-\right.$ is the cardinality of the set $\mathrm{M}_{1}$ ), which ends the proof of the above statement.

Statement 1 shows that if $t>0$, then deducting $t$ coordinates from the $M_{1}$ set of vector $\left[E\left(p_{1}^{s}\right), \ldots, E\left(p_{n}^{s}\right)\right]$ by one, then a vector, which balances the model, will be obtained. It creates the question, which of $t$ coordinates from the $M_{1}$ set, should deducted by one, in other words, to which of the consumers an additional unit of good should be assigned. The following solution is being proposed: increase the demand by one unit for those consumers who are the closest to its limits. This distance is determined by number of $x_{i}=\left(p_{i}+u_{i}-E\left(p_{i}^{s}\right)+1\right) u_{i}^{-1}$.

The $100 x_{i}$ number expresses the percentage of concession remaining $i^{\text {th }}$ consumer, if their demand will verify to the amount equal to $\mathrm{E}\left(p_{i}^{s}\right)-1$. In order to indicate a concrete consumer, to which an additional unit of good in $M_{1}$ set will be assigned, an ordering relations is introduced.

\section{Statement 2.}

If for every $i, j \in \mathrm{M}_{1}, i \mathrm{R} j \Leftrightarrow\left\{\begin{array}{ll}x_{i}<x_{j}, & x_{i} \neq x_{j} \\ i \geq j, & x_{i}=x_{j}\end{array}\right.$ then $\mathrm{R}$ relation is ordering relations in $\mathrm{M}_{1}$ set.

Proof

It should be presented that relation $\mathrm{R}$ is:

a) reversible, ie. for every $i \in \mathrm{M}_{1}$ and $\mathrm{R}_{i}$,

b) weakly symmetric, ie. for every $i, j \in \mathrm{M}_{1} i \mathrm{R} j$ and $j \mathrm{R} i \Leftrightarrow i=j$,

c) transitive, ie. for every $i, j, k \in \mathrm{M}_{1} i \mathrm{R} j$ and $j \mathrm{R} k \Leftrightarrow i \mathrm{R} k$,

d) consistent, ie. for every $i, j \in \mathrm{M}_{1} i \mathrm{R} j$ or $j \mathrm{R} i$.

Ad a) $i \mathrm{R} i$, because $i \geq i$ and $x_{i}=x_{i}$.

Ad b) $i \mathrm{R} j$, ie. $x_{i}<x_{j}$ or $\left(x_{i}=x_{j}\right.$ and $\left.i \geq j\right)$,

$j \mathrm{R} i$, ie. $x_{j}<x_{i}$ or $\left(x_{j}=x_{i}\right.$ and $\left.j \geq i\right)$.

If $i \mathrm{R} j$ and $j \mathrm{R} i$, then $x_{i}=x_{j}, i \geq j, j \geq i$, therefore $x_{i}=x_{j}$ and $i=j$.

Ad c) $i \mathrm{R} j$, therefore $x_{i}<x_{j}$ or $\left(x_{i}=x_{j}\right.$ and $\left.i \geq j\right)$,

$j \mathrm{R} k$, therefore $x_{j}<x_{k}$ or $\left(x_{j}=x_{k}\right.$ and $\left.j \geq k\right)$. 
If $x_{i} \neq x_{j} \neq x_{k}$, then $x_{i}<x_{j}<x_{k}$ or $x_{i}<x_{k}$, therefore $i \mathrm{R} k$.

If $x_{i}=x_{j} \neq x_{k}$, then $x_{i}=x_{j}<x_{k}$ or $x_{i}<x_{k}$, therefore $i \mathrm{R} k$.

If $x_{i} \neq x_{j}=x_{k}$, then $x_{i}<x_{j}=x_{k}$ or $x_{i}<x_{k}$, therefore $i \mathrm{R} k$.

If $x_{i}=x_{j}=x_{k}$, then $i \geq j$ and or $j \geq k$ or $i \geq k$.

Therefore $i \mathrm{R} k$.

Ad. d) Proof by contradiction.

If $i \mathrm{R} j$ does not occur, then $x_{j}>x_{i}$ or $\left(x_{j}=x_{i}\right.$ and $\left.i>j\right)$.

If $x_{i}>x_{j}$, a contradiction that $x_{j}>x_{i}$ or $x_{i}=x_{j}$.

If $x_{j}>x_{i}$, a contradiction that $x_{i}>x_{j}$ or $x_{j}=x_{i}$.

If $x_{i}=x_{j}$, a contradiction that $i=j$.

Must therefore take place one of the relations: $i \mathrm{R} j$ or $j \mathrm{R} i$, which ends the proof of the above statement.

Ordered $\mathrm{M}_{1}$ set, according $\mathrm{R}$ relations, determines by $\mathrm{M}_{1 \mathrm{R}}$. Increasing demand to the first $t^{\text {th }}$ consumers from the $\mathrm{M}_{1 \mathrm{R}}$ set, by one unit, leads to sustainable model. In addition, supply and demand of all contractors will be expressed by integers. It will be presented in the statement below.

\section{Statement 3.}

If $t>0, M_{1}^{+}=\left\{i \in \mathrm{M}_{1 \mathrm{R}}: i \leq s\right\}$,

$d_{i}=\left\{\begin{array}{ll}E\left(p_{i}^{s}\right)-1 & \text { for } i \in M_{1}^{+} \\ E\left(p_{i}^{s}\right) & \text { for } i \notin M_{1}^{+}\end{array}, \overrightarrow{\mathbf{d}}=\left(d_{1}, \ldots, d_{n}\right)\right.$

then: for $i \leq n d_{i}$ is an integer and $d_{1}+d_{2}+\ldots+d_{n}=0$.

Proof:

For $i \in M_{1}^{+} d_{i}$ is an integer as a difference of integers. For $i \notin M_{1}^{+} d_{i}$ is also an integer.

$$
\sum_{i=1}^{n} d_{i}=\sum_{i \in M_{1}^{+}} d_{i}+\sum_{i \notin M_{1}^{+}} d_{i}=\sum_{i \in M_{1}^{+}}\left(\mathrm{E}\left(p_{i}^{s}\right)-1\right)+\sum_{i \notin M_{1}^{+}} \mathrm{E}\left(p_{i}^{s}\right)=\sum_{i=1}^{n} \mathrm{E}\left(p_{i}^{s}\right)+\sum_{i \in M_{1}^{+}}(-1)=t-t=0
$$

\section{Conclusions}

Presented, in the above statement, the method of discrete vector correction is not just one, obviously, verification method of contractors' supply and demand. Having in mind various economic factors, the user can conduct a correction among any relation ordering the set of contractors, and among any assignment. Different measures of "distance" between vectors can also be used for this purpose. 


\section{References}

[1] Ladyga M., Tkacz M., The unsustainable production and consumption model, Polish Journal of Management Studies, vol. 4, Czestochowa University of Technology, 2011.

[2] Ladyga M., Tkacz M., Balancing method of unsustainable production and consumption model, Scientific Research of the Institute of Mathematics and Computer Science 2011, 2(10), 135-145.

[3] Ladyga M., Tkacz M., The properties of method balancing the unsustainable production and consumption model, Scientific Research of the Institute of Mathematics and Computer Science 2012, 3(11), 105-109.

[4] Ladyga M., Tkacz M., The explicitness of vector balancing the unsustainable production and consumption model, Polish Journal of Management Studies, vol. 5, Czestochowa University of Technology, 2012, 261-265. 